\title{
Dissociative recombination in reactive flows related to planetary atmospheric entries
}

\author{
Arnaud Bultel $^{1, \mathrm{a}}$, Julien Annaloro ${ }^{1, \mathrm{~b}}$ and Marie-Claude Druguet ${ }^{2, \mathrm{c}}$ \\ ${ }^{1}$ CORIA, UMR CNRS 6614, Université de Rouen, Site Universitaire du Madrillet, BP. 12, \\ 76801 Saint-Etienne du Rouvray, France \\ ${ }^{2}$ Aix-Marseille Université, CNRS, IUSTI UMR 7343, 13013 Marseille, France
}

\begin{abstract}
The Dissociative Recombination (DR) processes play a significant role in plasma chemistry. This article illustrates this role from the modeling point of view in the case of reactive flows related to atmospheric entry plasmas. Two situations are investigated, for which the studied plasma is nitrogen. The first configuration corresponds to the relaxation process behind a strong shock wave moving at high Mach number in a shock tube, the second one to the recombination taking place in an expanding plasma flowing in a diverging nozzle. In both cases, the collisional-radiative model CoRaM- $\mathrm{N}_{2}$, involving $\mathrm{N}_{2}, \mathrm{~N}, \mathrm{~N}_{2}^{+}, \mathrm{N}^{+}$and electrons, is implemented in an Eulerian 1D code able to compute the aerodynamic fields; calculations are performed in standard conditions. We show that, according to the rate coefficients used for the DR processes, the population density of the charged species especially $\mathrm{N}_{2}^{+}$is strongly modified only for the post-shock flow.
\end{abstract}

\section{Introduction}

During the entry of a probe into the upper layers of a planetary atmosphere, the flow relative to the probe is hypersonic and the Mach number can reach a value of 40. In this context, the flow is strongly slowed down at the probe surface because of the gas compression in a shock layer where the translation temperature of the flow increases owing to the conversion kinetic energy $\rightarrow$ internal energy $[1,2]$. The characteristic time of this conversion is very short so that the flow is in thermochemical non equilibrium [3]. The temperature in the shock layer region is of the order of $30000 \mathrm{~K}$. As a result, dissociation and ionization elementary processes take place and lead to the gas $\rightarrow$ plasma transition. In order to correctly predict the properties of the shock layer flow and particularly assess the radiative fluxes impinging the probe surface, a complete characterization of the atomic and molecular excited states population densities is mandatory.

The related chemistry can be experimentally studied in ground test facilities such as plasma wind tunnels, shock tubes or nozzle setups [4]. Shock tubes can be used to study the dissociation-ionization

\footnotetext{
a e-mail: arnaud.bultel@coria.fr

b e-mail: julien.annaloro@coria.fr

c e-mail: marie-claude.druguet@univ-amu.fr; 5 rue Enrico Fermi
}

This is an Open Access article distributed under the terms of the Creative Commons Attribution License 4.0, which permits unrestricted use, distribution, and reproduction in any medium, provided the original work is properly cited. 
Table 1. Species and states considered in CoRaM- $\mathrm{N}_{2}$.

\begin{tabular}{ll} 
Species & States \\
\hline $\mathrm{N}_{2}$ & $X^{1} \Sigma_{g}^{+}(v=0 \rightarrow 67), A^{3} \Sigma_{u}^{+}, B^{3} \Pi_{g}, W^{3} \Delta_{u}, B^{\prime 3} \Sigma_{u}^{-}, a^{\prime 1} \Sigma_{u}^{-}, a^{1} \Pi_{g}, w^{1} \Delta_{u}, G^{3} \Delta_{g}, C^{3} \Pi_{u}, E^{3} \Sigma_{g}^{+}$ \\
$\mathrm{N}_{2}^{+}$ & $X^{2} \Sigma_{g}^{+}, A^{2} \Pi_{u}, B^{2} \Sigma_{u}^{+}, a^{4} \Sigma_{u}^{+}, D^{2} \Pi_{g}, C^{2} \Sigma_{u}^{+}$ \\
$\mathrm{N}^{+}$ & ${ }^{4} S,{ }^{2} D,{ }^{2} P,{ }^{4} P_{1 / 2}, \ldots .(63$ states $)$ \\
$\mathrm{N}^{+}$ & ${ }^{3} P_{0},{ }^{3} P_{1},{ }^{3} P_{2},{ }^{1} D_{2},{ }^{1} S_{0},{ }^{5} S_{2}^{o},{ }^{3} D_{3}^{o},{ }^{3} D_{2}^{o},{ }^{3} D_{1}^{o}$ \\
$e^{-}$ & -
\end{tabular}

just behind the shock wave, and plasmas wind tunnels and nozzles are more relevant for recombination situations like in the vicinity of the probe surface. Since the beginning of the research activities related to spatial flights, nitrogen has been considered as a benchmark, because of its significant relative density in the atmosphere of Earth or Titan, and its presence in Mars atmosphere. Since then, the chemistry of $\mathrm{N}_{2}$ has been widely studied from the experimental point of view; it is therefore the main subject of the present study.

A relevant way of identifying the chemistry of $\mathrm{N}_{2}$ is to compare experimental results with those resulting from modeling approaches. The latter are developed by considering excited states-dependent forward and backward elementary processes, the rate coefficient of which being related to each other by the detailed balance principle. For ionization situations, Associative Ionization (AI) such as $\mathrm{N}+\mathrm{N} \rightarrow$ $\mathrm{N}_{2}^{+}+$e- plays a role in the production of the first free electrons in pure nitrogen plasma flows. In the case of recombination situations like those taking place in nozzles, the Dissociative Recombination (DR) $\mathrm{N}_{2}^{+}+$e- $\rightarrow \mathrm{N}+\mathrm{N}$ plays a role in the evolution of electron density. Reactive and product species involved in these processes can be electronically excited. Therefore, the excited states have to be taken into account explicitly. State-to-state models are thus relevant tools for the thorough study of chemical mechanisms. Since these excited states are also coupled to radiative processes, Collisional-Radiative (CR) models are therefore required.

In the present paper, we propose to focus our attention on the influence of DR (therefore of AI by the detailed balance) on the chemistry of $\mathrm{N}_{2}$ (1) in ionization situations such as post-shock relaxations in bow shock layers or behind normal shock waves in shock tubes and (2) in recombination situations studied in diverging nozzles. The CR model CoRaM- $\mathbf{N}_{2}$ is used to assess the influence of DR on the reactive gas composition. The model itself and its implementation in a 1D Eulerian code are described in Sect. 2. Then, results obtained in ionization or recombination situations are analyzed in Sect. 3.

\section{Implementation of the CR model CoRaM-N 2}

The Collisional-Radiative model CoRaM-N $\mathbf{N}_{2}$ has already been presented in earlier papers so that only its main characteristics are given below. The interested reader will find all details in $[5,6]$ mainly.

This CR model takes into account free electrons and the four species $\mathrm{N}_{2}, \mathrm{~N}_{2}^{+}, \mathrm{N}$ and $\mathrm{N}^{+}$with a total of 156 individual vibrational and electronic states summarized in Table 1 . These excited states cover a large spectrum of energy so that with this model it is possible to calculate situations corresponding to high specific enthalpy. It is interesting to note that the model is not only electronic specific but also vibrational specific since vibrationally excited $\mathrm{N}_{2}$ molecules in their electronic ground state are considered as full species in the present case. This is particularly appropriate for modeling strong non equilibrium dissociation configurations for which the concept of vibrational temperature is irrelevant.

These different states are considered as full species and are populated according to the following different elementary processes:

- vibrational processes (vibrational excitation under electron, $\mathrm{N}_{2}$ and $\mathrm{N}$ impact) [7-9],

- dissociation of electronic excited states of $\mathrm{N}_{2}$ and of all electronic states of $\mathrm{N}_{2}^{+}$under electron impact [10],

- electronic excitation under electron, $\mathrm{N}_{2}$ and $\mathrm{N}$ impact [3, 10, 11, 13-16, 18], 
Table 2. Branching ratios considered in the present work (Peterson et al. [20]).

\begin{tabular}{lcl} 
Branching ratio & Percentage of the total DR & Rate coefficient $\left(\mathrm{m}^{3} \mathrm{~s}^{-1}\right)$ \\
\hline $\mathrm{N}\left({ }^{4} S\right)+\mathrm{N}\left({ }^{2} D\right)$ & 37 & $6.48 \times 10^{-14}\left(\frac{T_{e}}{800}\right)^{-0.3}$ \\
$\mathrm{~N}\left({ }^{4} S\right)+\mathrm{N}\left({ }^{2} P\right)$ & 11 & $1.93 \times 10^{-14}\left(\frac{T_{e}}{800}\right)^{-0.3}$ \\
$\mathrm{~N}\left({ }^{2} D\right)+\mathrm{N}\left({ }^{2} D\right)$ & 52 & $9.10 \times 10^{-14}\left(\frac{T_{e}}{800}\right)^{-0.3}$ \\
\hline
\end{tabular}

- excitation transfer $[11,17,18]$,

- ionisation under electron, $\mathrm{N}_{2}$ and $\mathrm{N}$ impact $[3,10,11,14-16,19]$,

- charge exchange [18] and

- dissociative recombination [20].

It is important to note for the upcoming discussion that the DR rate coefficients used in the present work have been measured by Peterson et al. on the ion storage ring CRYRING [20] with a certain vibrational excitation close to the one calculated in the present context. Using their data is therefore particularly appropriate. These processes involve the ground ${ }^{4} S$ and the two metastable ${ }^{2} D$ and ${ }^{2} P$ states of $\mathrm{N}$ after recombination. Their branching ratios have also been experimentally determined by Peterson et al. for which the corresponding data is reproduced in Table 2.

The backward collisional elementary processes (including the three-body recombination which competes with the DR processes) are also implemented in CoRaM-N $\mathbf{N}_{2}$ where each related rate coefficient is derived from the forward rate coefficient and from the corresponding equilibrium constant using the detailed balance principle. Thus, the set of processes described above represents a total of about 40000 forward and backward elementary processes and is better detailed in [21].

These elementary collisional processes take place simultaneously with elementary radiative processes, the list of which is given in $[5,6]$. The possible self-absorption of radiation by the flow is taken into account through the escape factor due to Holstein [22] for which a Doppler-type broadening has been assumed.

The previous collisional and radiative elementary processes are implemented in the source term of the (species, momentum and energy) balance equations relevant for the flows studied here. Since these flows are characterized by high speed and weakly diverging geometries, they can be modeled by the 1D Euler equations. Note that we assume that the density within the flow is weak. Then, the perfect gas assumption is justified and the Dalton's law can be applied. The resulting balance equations are not given here since such details are beyond the scope of the present paper. Let us only mention that the low density of the flow prevents the thermal equilibrium: electrons and heavy particles are characterized by different translation temperatures written $T_{e}$ and $T_{A}$, respectively. The interested reader will find the mathematical form of these equations in $[6,23]$.

\section{Results for ionization or recombination situations}

\subsection{Post-shock relaxation at high Mach number}

The first situation studied corresponds to the flow relaxing behind the bow shock wave that is detached from the surface of an entering body. This configuration is similar to the one observed in shock tubes behind the shock front by a simple change of the coordinate system. In the present work, the shock front itself is not resolved numerically but it is considered as a discontinuity across which momentum and energy are conserved, the chemistry being frozen. Rankine-Hugoniot equations are therefore used. The subsequent relaxation is the result of the $T_{A}$ discontinuity due to the sudden change of density across the shock wave.

The flow conditions chosen for the present work correspond to the well-known FIRE II flight experiment reported in [24]. We adopt representative upstream conditions for which the Mach number, the pressure and the temperature are $\mathcal{M}=33, p=33 \mathrm{~Pa}$ and $T_{A}=250 \mathrm{~K}$, respectively. 


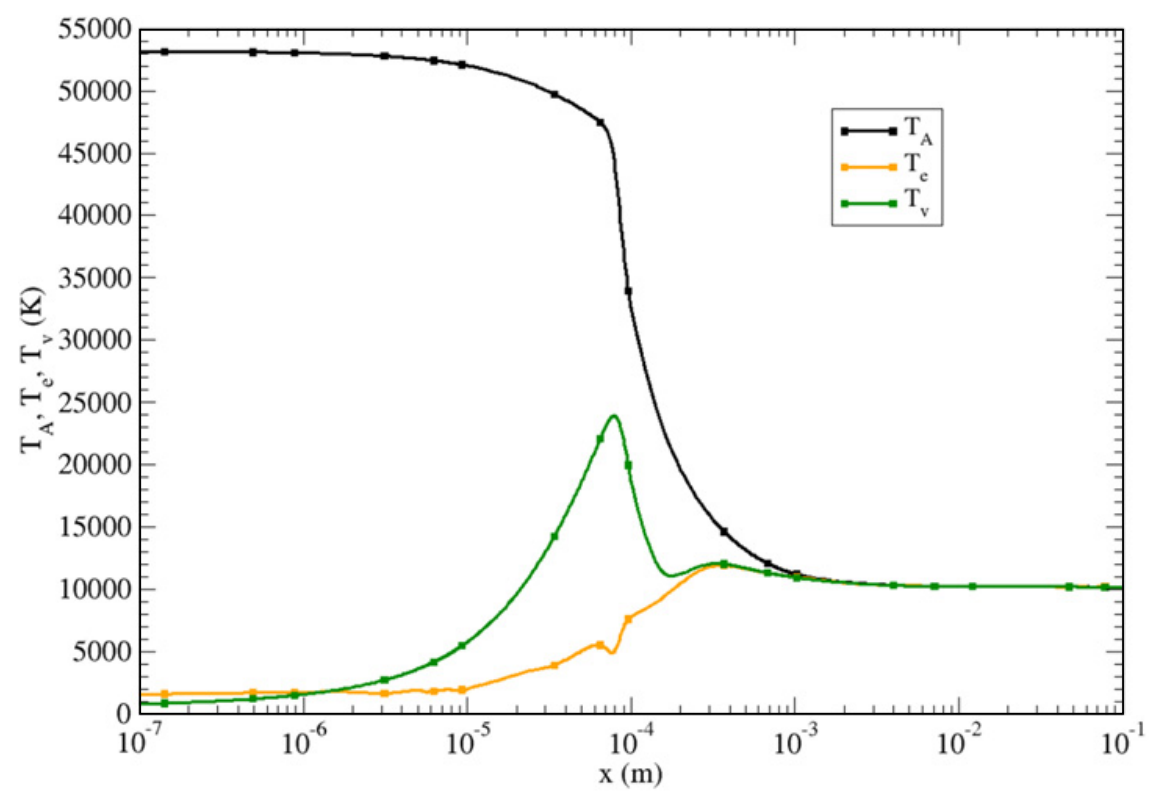

Figure 1. Spatial evolution of the heavy particles temperature $T_{A}$, the electron temperature $T_{e}$ and the vibrational temperature $T_{v}$ behind the shock front for the typical FIRE II flight experiment upstream conditions $\mathcal{M}=33$, $p=33 \mathrm{~Pa}$ and $T_{A}=250 \mathrm{~K}$.

Figure 1 illustrates the spatial evolution of the flow temperatures from $x=0$ where the shock front is located. We see that just behind the shock front $T_{A}$ reaches a high value of around $55000 \mathrm{~K}$ resulting from the transfer of kinetic energy to thermal energy through the shock wave. Then, the heavy-particle temperature, $T_{A}$, decreases because of the vibrational processes that take place and lead to the vibrational excitation as revealed by the increase in $T_{v}$. Note that this vibrational temperature is post-processed since the $\mathrm{N}_{2}$ molecules on their vibrational levels are considered as full species. In the present case, $T_{v}$ is the excitation temperature of the first five vibrational levels of the electronic ground state of $\mathrm{N}_{2} . T_{v}$ reaches a maximum while $T_{e}$ keeps increasing. Note that a slight decrease in $T_{e}$ is observed when $T_{v}$ reaches its maximum. This slight effect is the result of the significant value of $T_{v}$ and the pumping of electron energy resulting from the very good coupling between electrons and vibrational states. A first excitation equilibrium state takes place around $3 \times 10^{-4} \mathrm{~m}$ where $T_{v} \approx T_{e}$ is observed. Finally, $T_{A}$ couples with the other temperatures of the flow around $10^{-3} \mathrm{~m}$, which means that the local thermodynamic equilibrium is reached. Behind that equilibrium point, the temperature is slightly decreasing owing to the radiative losses.

Figure 2 illustrates the spatial evolution of the population densities behind the shock wave in the same conditions. We can note the progressive vibrational excitation in correlation with the dissociation processes shown by the strong increase of $\mathrm{N}$ density. Electron density increase is as strong as the one observed for the $\mathrm{N}$ density, but is spatially shifted. Therefore, the flow "history" is divided into the successive three phases vibrational excitation $\rightarrow$ dissociation $\rightarrow$ ionization.

The $\mathrm{N}_{2}^{+}$density undergoes an increase similar to the one observed for the electron density, but with a lower value than for $\mathrm{N}^{+}$. This means that electroneutrality is mainly ensured by $\mathrm{N}^{+}$ions. $\mathrm{N}_{2}^{+}$ions are dissociated from $10^{-4} \mathrm{~m}$ and become negligible with respect to $\mathrm{N}^{+}$in terms of density.

In this context, it is interesting to study specifically the influence of the DR (therefore the AI) processes, since these processes involve $\mathrm{N}, \mathrm{N}_{2}^{+}$and electrons. Figure 3 illustrates the spatial profiles obtained for $\mathrm{N}_{2}^{+}$when the DR processes are removed from $\mathbf{C o R a M}-\mathbf{N}_{2}$. Before $10^{-4} \mathrm{~m}$, the AI processes 


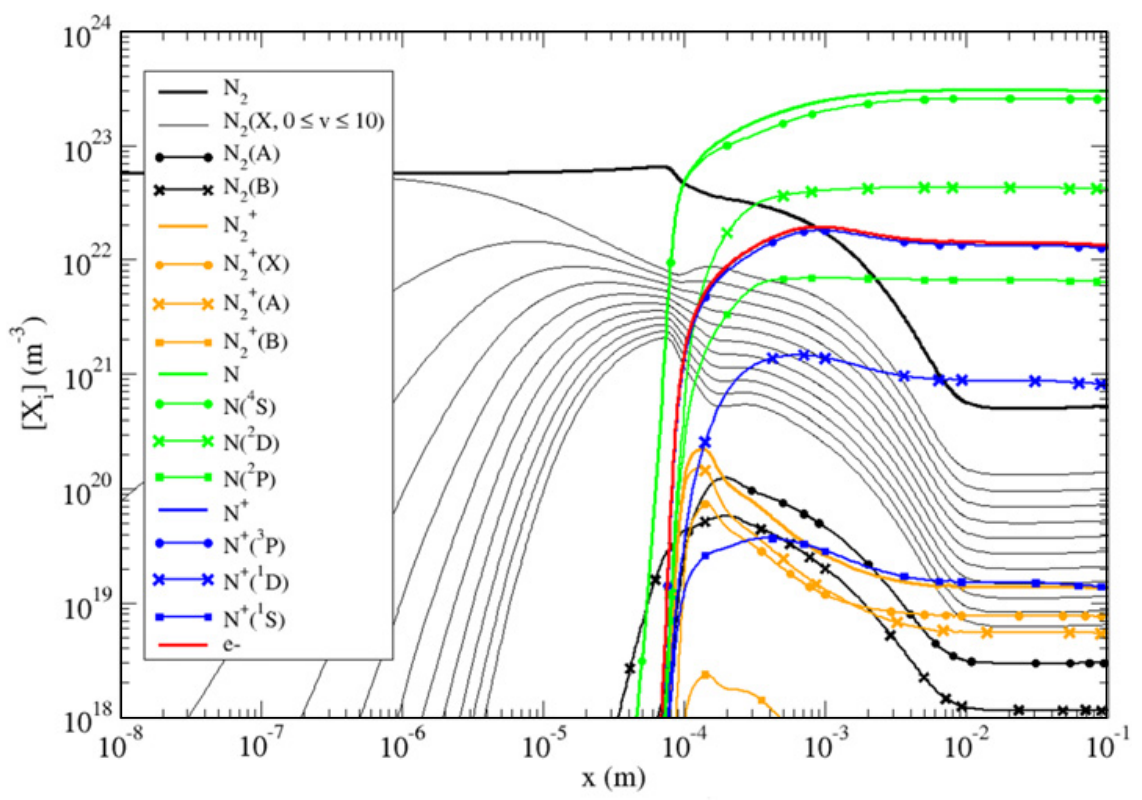

Figure 2. Same as Fig. 1 but for the population densities. Note that the main ion $\left(\mathrm{N}^{+}\right)$density and electron density lines are superimposed due to electroneutrality.

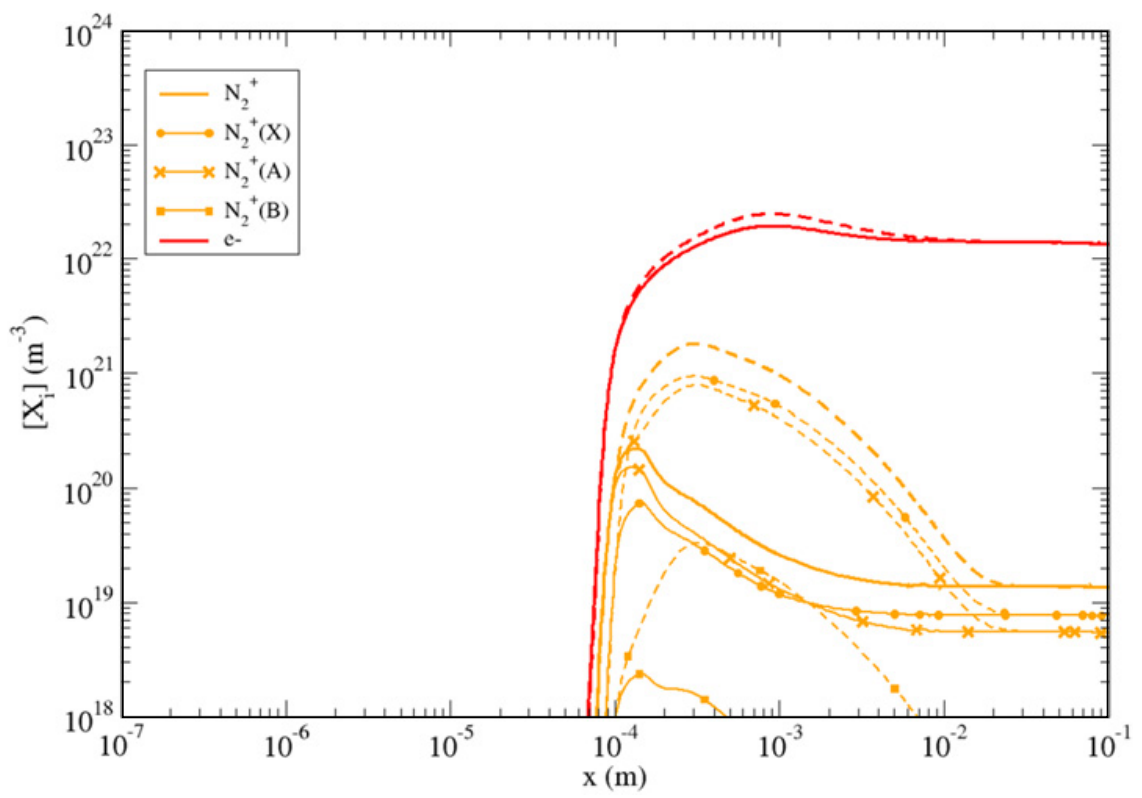

Figure 3. Same as Fig. 2 but for $\mathrm{N}_{2}^{+}$ions total density, ground and first two excited electronic states, and electron density (solid lines: with DR processes, dashed lines: without DR processes). 


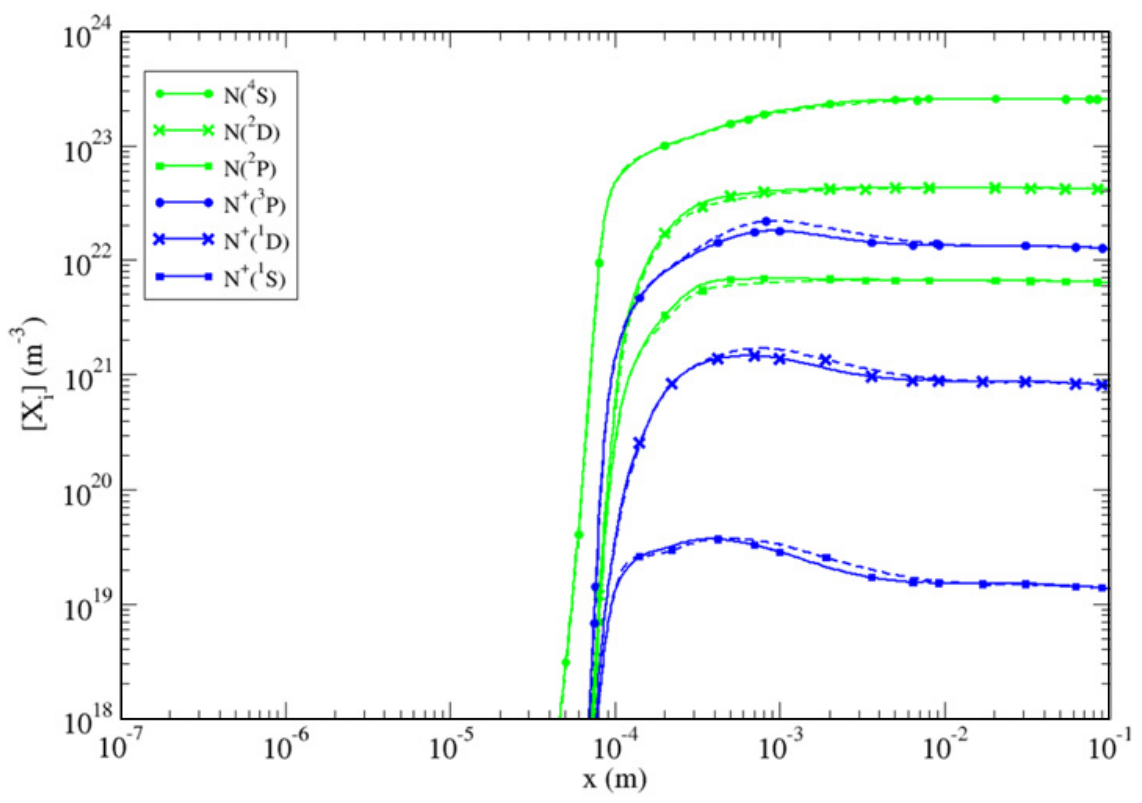

Figure 4. Same as Fig. 3 but for the states of $\mathrm{N}$ and $\mathrm{N}^{+}$(solid lines: with DR processes, dashed lines: without DR processes).

drive the production of the first electrons (this cannot be shown on Fig. 3). Beyond $10^{-4} \mathrm{~m}$, the $\mathrm{N}_{2}^{+}$ density is strongly increased when DR processes are removed. The $\mathrm{N}_{2}^{+}$density can be reduced by a factor of 40 around $10^{-3} \mathrm{~m}$ when DR and AI processes occur. Electron density is much higher than $\mathrm{N}_{2}^{+}$ density. As a result, electron density is weakly modified by the value of the rate coefficient used for the DR processes. Indeed, Fig. 3 shows that electron density is increased by only a factor of $25 \%$ without DR processes.

As illustrated by Fig. 2, the dissociation degree of the plasma is high beyond $10^{-4} \mathrm{~m}$. Therefore, we expect a weak influence of the DR processes on the density of the states of $\mathrm{N}$. This is confirmed by Fig. 4 where the spatial profiles of the number densities of $\mathrm{N}\left({ }^{4} S\right), \mathrm{N}\left({ }^{2} D\right)$ and $\mathrm{N}\left({ }^{2} P\right)$ are displayed. We see that removing the DR processes leads to a weak decrease in the density of $\left.\mathrm{N}^{2} D\right)$ and $\mathrm{N}\left({ }^{2} P\right)$, whereas the density of $\mathrm{N}\left({ }^{4} S\right)$ is not significantly modified. Although most of the DR processes produce $\mathrm{N}\left({ }^{2} D\right)$ as illustrated by the branching ratios displayed in Table 2 , the density of $\mathrm{N}\left({ }^{2} D\right)$ results mainly from the electron-induced excitation from the ${ }^{4} S$ ground state. Therefore the influence of the DR on the population density of $\left.\mathrm{N}^{2} D\right)$ is weak.

Nevertheless, this influence propagates from one species to another through the coupling resulting from the chemical mechanism. Even if the $\mathrm{N}$ density is not basically modified, the $\mathrm{N}_{2}$ states population densities present significant changes due to the high dissociation degree observed downstream, far from the ionization zone. Indeed, Fig. 5 shows that the vibrational states of $\mathrm{N}_{2}(X)$ and the first two electronic excited states A and B are more populated when the DR processes are removed. This is due to the decrease in the $\mathrm{N}$ density. The departure from the case considering the DR processes can reach a ratio of 3 for the vibrational ground state around $10^{-2} \mathrm{~m}$.

As a conclusion, the DR and AI (by detailed balance) processes present a significant influence on the density of the $\mathrm{N}_{2}^{+}$states for the flow conditions considered in the present section. Even if $\mathrm{N}_{2}^{+}$is not a major species in the mixture, its coupling with the other species through the elementary processes leads to changes of the population densities easy to observe. 


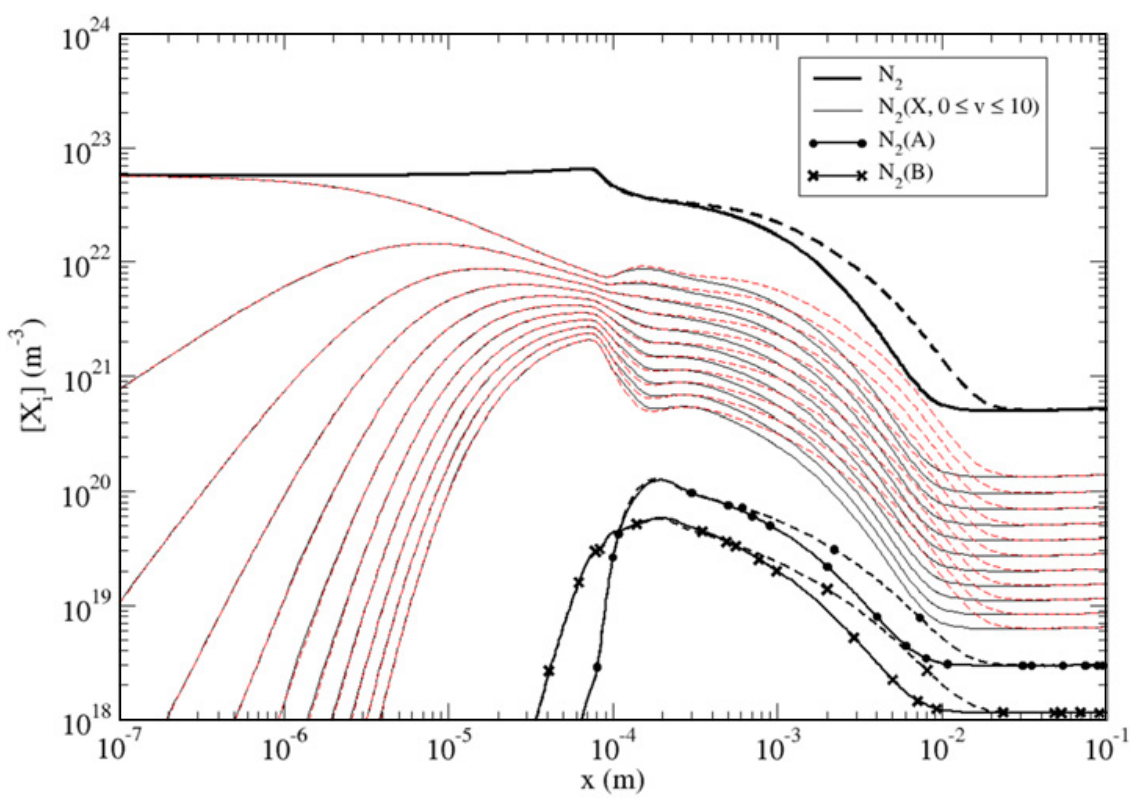

Figure 5. Same as Fig. 3 but for the states of $\mathrm{N}_{2}$ (solid lines: with DR processes, dashed lines: without DR processes, in red dashed lines: vibrational states of $\mathrm{N}_{2}$ without DR processes).

\subsection{Supersonic relaxation in diverging nozzle}

In Sect. 3.1, we have put forward the sensitivity of the vibrational distribution to the rate coefficients of the DR processes. Diverging nozzles are often used to further characterize the behavior of this distribution when recombination situations occur. Since the dissociation degree is influenced by the DR, we expect the flow characteristics to be sensitive to the DR rate coefficient, for this type of flow.

Figure 6 illustrates the spatial profiles obtained for $T_{e}$ and $T_{A}$ using $\mathbf{C o R a M - \mathbf { N } _ { 2 }}$ to model the wellknown Ohio State University diverging nozzle [23] in typical conditions (reservoir at $10^{6} \mathrm{~Pa}$ and $\mathrm{T}=$ $6000 \mathrm{~K}$ ). The flow is in thermochemical equilibrium at $T_{A}=T_{e}=5000 \mathrm{~K}$ at the nozzle throat (located at $x=0$ ) owing to the reservoir-throat converging connection. Downstream, the flow departs from thermal equilibrium for $x>10^{-5} \mathrm{~m}$ : $T_{e}$ stabilizes at $4000 \mathrm{~K}$ whereas $T_{A}$ decreases down to $700 \mathrm{~K}$ near the nozzle exit. The decrease in $T_{A}$ is due to the work losses resulting from the divergence of the nozzle. The decrease in $T_{e}$ is due to the coupling with the vibrational distribution through the vibrational elementary processes involving electrons. These processes are progressively frozen owing to the collapse of collision frequency resulting from the opening of the nozzle.

For the same conditions, Fig. 7 displays the spatial profiles of the population density of the main atomic and molecular states along the nozzle axis. We can observe their decrease starting at $10^{-3} \mathrm{~m}$ from the nozzle throat. Between the throat and $x=0.5 \mathrm{~m}$, the density ratio $[X(0.5 \mathrm{~m})] /[X(0)]$ for $X \equiv$ $\mathrm{N}$ or $\mathrm{N}_{2}$ is the same as for the total density, which means that the recombination from atoms to molecules is not significant. By contrast, this ratio is largely higher for ions and excited states. The recombination from ions to atoms or molecules is therefore significant. It is interesting to note that electrons and $\mathrm{N}_{2}^{+}$ions have the same density in the present case. Therefore we expect a higher influence of the DR processes on the behavior of the charged particles.

In order to assess this influence, we have performed calculations with rate coefficients listed in Table 2 turned down to 0 . These conditions do not modify the profiles of $T_{e}$ and $T_{A}$ displayed in Fig. 6 . Except for $\mathrm{N}^{+}, \mathrm{N}_{2}^{+}$and electrons, the population density profiles are not modified as illustrated by Fig. 7 . 


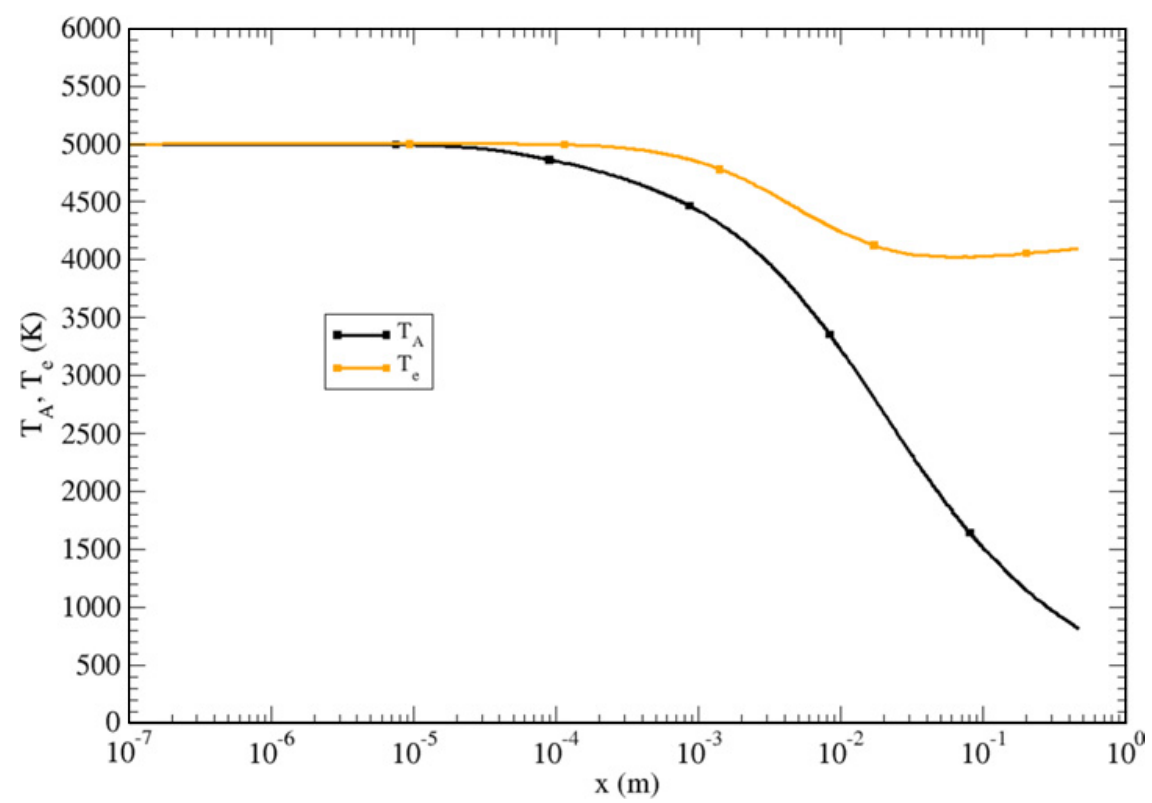

Figure 6. Spatial profiles of $T_{A}$ and $T_{e}$ in the diverging part of the Ohio State University nozzle [23] for the reservoir conditions $10^{6} \mathrm{~Pa}$ and $\mathrm{T}=6000 \mathrm{~K}$. The throat is located at $x=0$.

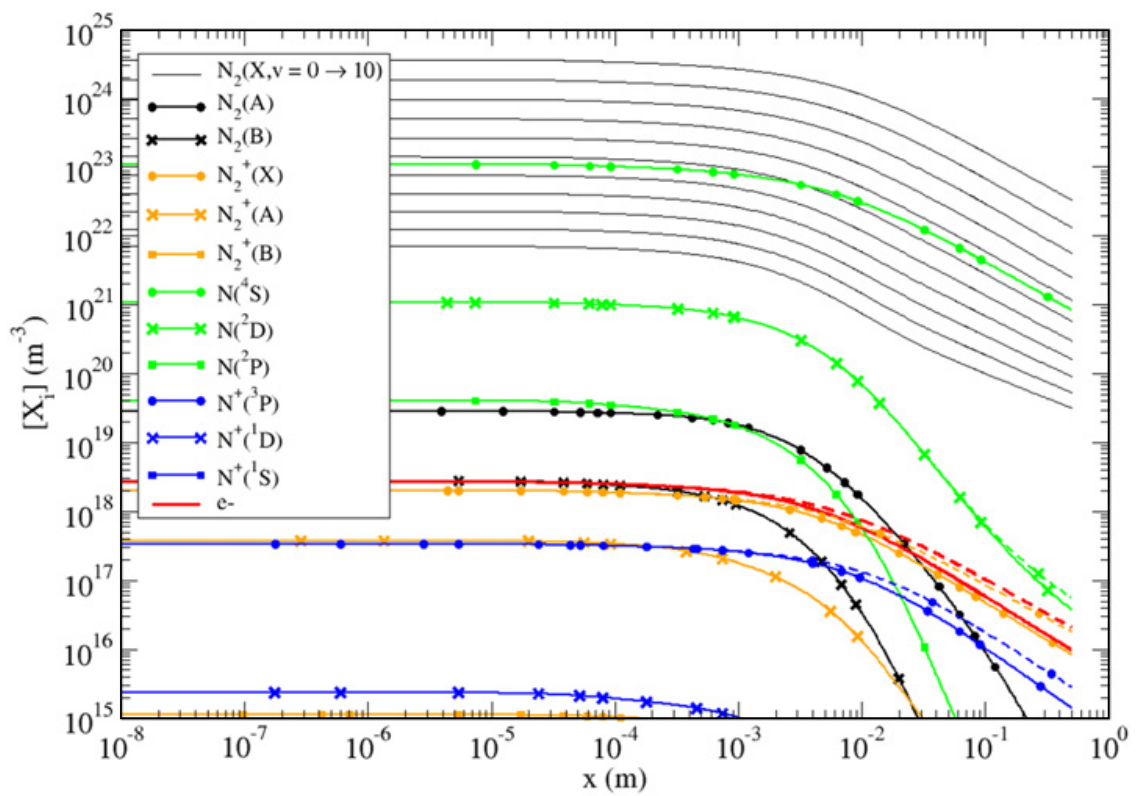

Figure 7. Same as Fig. 2, but for the density profiles in the conditions of Fig. 6. When the DR processes are removed from CoRaM- $\mathbf{N}_{2}$, only $\mathrm{N}^{+}, \mathrm{N}_{2}^{+}$and electrons are modified (the corresponding curves are displayed with dashed lines). 
Without DR, the recombination is weaker which leads to a higher electron density at the nozzle exit. The $\mathrm{N}_{2}^{+}$density is then higher. The electron and $\mathrm{N}_{2}^{+}$densities are multiplied by a factor of 2 when DR processes are removed. The $\mathrm{N}^{+}$density is multiplied by the same factor owing to its coupling with the $\mathrm{N}_{2}^{+}$density mainly through the charge exchange elementary processes.

\section{Conclusion}

In this article, we have brought into light the sensitivity of the behavior of nitrogen thermochemical non-equilibrium flows related to planetary entry configurations to the Dissociative Recombination (DR) using the Collisional-Radiative model CoRaM-N $\mathbf{N}_{2}$. Generally speaking, these flows involve high temperature levels so that the dissociation degree is strong and the molecular ions density is low. The influence of the DR processes is therefore expected to be relatively weak. However, this is not what is shown by the present study. In dissociation-ionization situations behind strong shock waves, we have shown that the DR processes play a significant role in the behavior of the charged species. By contrast, this influence is weaker in recombination situations along diverging nozzles in the chosen conditions. In these circumstances, comparisons between experimental and theoretical results may lead to alternative determination of the rate coefficients of the DR processes. Thus, interesting comparisons may be performed at high temperature with the rate coefficients measured at low temperature in the ion storage ring CRYRING. In particular, the influence of the rovibrational excitation of $\mathrm{N}_{2}^{+}$on the DR processes may be estimated.

The research leading to these results has received funding from the French Spatial Agency CNES and from the European Community's Seventh Framework Programme (FP7/2007-2013) under grant agreement No. 242311.

\section{References}

[1] Anderson, J. D., Hypersonic and High Temperature Gas Dynamics (McGraw-Hill Book Company, New York, 1989)

[2] Druguet, M.-C., Shock Waves 20 (2010) 251-261

[3] Bultel, A., Chéron, B. G., Bourdon, A., Motapon, O., and Schneider, I. F., Phys. Plasmas 13 (2006) 043502 (11pp)

[4] Boubert, P., Bultel A., Chéron B.G., and Vervisch, P., J. Tech. Phys. 50 (2009) 163-179

[5] Bultel, A., and Annaloro, J., Plasma Sources Sci. Technol. 22 (2013) 025008 (17pp)

[6] Annaloro, J., Bultel, A., and Omaly, P., J. Thermophys. Heat Transfer 28 (2014) 608

[7] Laporta, V., Celiberto, R., and Wadhera, J. M., Plasma Sources Sci. Technol. 21 (2012) 055018 (10pp)

[8] Armenise, I., Capitelli, M., Colonna, G., and Gorse, C., Journal of Thermophys. Heat Transfer 10 (1996) 397-405

[9] Esposito, F., Armenise, I., and Capitelli, M., Chem. Phys. 331 (2006) 1-8

[10] Teulet, P., Sarrette, J.-P., and Gomes, A.-M., J. Quant. Spectrosc. Radiat. Transfer 62 (1999) 549569

[11] Capitelli, M., Ferreira, C. M., Gordiets, B. F., and Osipov, A. I., Plasma Kinetics in Atmospheric Gases (Springer-Verlag, Berlin, 2000)

[12] Lino da Silva, M., Guerra, V., and Loureiro, J., Chem. Phys. 342 (2007) 275-287

[13] Park, C., AIAA Meeting Papers (2008) 2008-1446

[14] Drawin, H. W., Collision and Transport Cross Sections (1966) EUR-CEA-FC 383

[15] Park, C., Journal of Thermophysics 2 (1988) 8-16

[16] Lotz, W., Zeitschrift für Phyzik 216 (1968) 241-247 
[17] Starik, A. M., Titova, N. S., and Arsentiev, I. V., Plasma Sources Sci. Technol. 19 (2010) 015007 (23pp)

[18] Kossyi, I. A., Kostinsky, A. Yu., Matveyev, A. A, and Silakov, V. P., Plasma Sources Sci. Technol. 1 (1992) 207-220

[19] Kim, Y. K., and Desclaux, J.-P., Phys. Rev. A 66 (2002) 012708 (12pp)

[20] Peterson, J. R., Le Padellec, A., Danared, H., Dunn, G. H., Larsson, M., Larson, A., Peverall, R., Stromholm, C., Rosen, S., af Ugglas, M., and van der Zande, W. J., The Journal of Chemical Physics 108 (1998) 1978-1988

[21] Annaloro, J., "Modèles collisionnels-radiatifs appliqués aux situations d'entrée atmosphérique martienne et terrestre," Ph.D. Dissertation (in French), CORIA, Université de Rouen, France (2013)

[22] Holstein, T., Phys. Rev. 72 (1947) 1212-1233

[23] Babu, V., and Subramaniam, V. V., J. Thermophys. Heat Transfer 9 (1995) 227-232

[24] Cornette, E. S., Forebody Temperatures and Calorimeter Heating Rates Measured during Project FIRE II Reentry at 11.35 Kilometers per Second (1966) NASA TM-X-1305 